\title{
A FINITE ELEMENT DISCRETIZATION OF THE THREE-DIMENSIONAL NAVIER-STOKES EQUATIONS WITH MIXED BOUNDARY CONDITIONS
}

\author{
Christine Bernardi ${ }^{1}$, Frédéric Hecht ${ }^{1}$ And RÜDiger Verfürth ${ }^{2}$
}

\begin{abstract}
We consider a variational formulation of the three-dimensional Navier-Stokes equations with mixed boundary conditions and prove that the variational problem admits a solution provided that the domain satisfies a suitable regularity assumption. Next, we propose a finite element discretization relying on the Galerkin method and establish a priori and a posteriori error estimates.
\end{abstract}

Mathematics Subject Classification. 65N30, 65N15, 65J15.

Received December 1st, 2008. Revised May 25, 2009.

Published online August 21, 2009.

\section{INTRODUCTION}

Let $\Omega$ be a bounded connected domain in $\mathbb{R}^{3}$, with a Lipschitz-continuous boundary $\partial \Omega$. We consider a partition without overlap of $\partial \Omega$ into two connected parts $\Gamma_{m}$ and $\Gamma$ and introduce the unit outward normal vector $\mathbf{n}$ to $\Omega$ on $\partial \Omega$. We are interested in the finite element discretization of the Navier-Stokes equations, for a positive constant viscosity $\nu$,

$$
\begin{array}{rlrl}
-\nu \Delta \mathbf{u}+(\mathbf{u} \cdot \nabla) \mathbf{u}+\operatorname{grad} P & =\mathbf{f} & & \text { in } \Omega, \\
\operatorname{div} \mathbf{u}=0 & & \text { in } \Omega, \\
\mathbf{u}=\mathbf{0} & & \text { on } \Gamma, \\
\mathbf{u} \cdot \mathbf{n}=0 & & \text { on } \Gamma_{m}, \\
(\operatorname{curl} \mathbf{u}) \times \mathbf{n}=\mathbf{0} & & \text { on } \Gamma_{m},
\end{array}
$$

where the unknowns are the velocity $\mathbf{u}$ and the pressure $P$ of the fluid. This type of mixed boundary conditions appears in a large number of physical situations, the simplest one being a tank closed by a membrane on a part of its boundary (the index " $m$ " in $\Gamma_{m}$ means membrane). An other example is the flow in a bifurcating pipe [8].

The Navier-Stokes equations with mixed boundary conditions have been considered for a long time. We refer to [4] for a pioneering paper on this subject. However, in a number of works concerning these mixed conditions, the boundary of the domain $\Omega$ is assumed to be piece-wise smooth. Here, we prove the existence of a solution

\footnotetext{
Keywords and phrases. Three-dimensional Navier-Stokes equations, mixed boundary conditions, finite element methods, a priori error estimates, a posteriori error estimates.

${ }^{1}$ Laboratoire Jacques-Louis Lions, C.N.R.S. \& Université Pierre et Marie Curie, B.C. 187, 4 place Jussieu, 75252 Paris Cedex 05, France. bernardi@ann.jussieu.fr; hecht@ann.jussieu.fr

${ }^{2}$ Ruhr-Universität Bochum, Fakultät für Mathematik, 44780 Bochum, Germany. ruediger.verfuerth@ruhr-uni-bochum.de
} 
to problem (1.1) with minimal regularity assumptions on the boundary. We refer to [2] for similar results which, however, mainly concern the two-dimensional situation.

In order to discretize the Navier-Stokes equations provided with the boundary conditions on $\Gamma_{m}$, a new formulation has been recently introduced in [20] (see also [11,12]) and has been extended to mixed conditions in [1]: the vorticity of the fluid is considered as a third independent unknown. However, we prefer to keep the formulation with two unknowns, in order to make use of standard finite element results. We propose several finite element discretizations of our problem and prove optimal a priori estimates provided some regularity assumptions are satisfied. These conditions seem to be not too restrictive. In addition, we establish $a$ posteriori error estimates which are optimal except in a neighbourhood of the re-entrant corners and edges in $\Gamma_{m}$. Despite this lack of optimality, we think that the discretizations that we propose lead to efficient simulations of incompressible viscous fluids in realistic situations.

An outline of the paper is as follows:

- In Section 2, we give a variational formulation of the problem and prove the existence of a solution.

- Section 3 is devoted to the description of the finite element discretization.

- In Section 4, we prove the existence of a solution for the discrete problem and derive a priori error estimates.

- In Section 5, finally, we present a posteriori error estimates based on residual error indicators.

- A numerical experiment is described in Section 6.

\section{The Continuous PRoblem}

From now on, we assume for simplicity that $\Omega$ is simply-connected and has a connected boundary, and also that $\partial \Gamma_{m}=\partial \Gamma$ is a Lipschitz-continuous submanifold of $\partial \Omega$. For the variational formulation of problem (1.1), we first observe by using the formulas

$$
-\Delta \mathbf{u}=\operatorname{curl}(\operatorname{curl} \mathbf{u})-\operatorname{grad}(\operatorname{div} \mathbf{u})
$$

and

$$
(\mathbf{u} \cdot \nabla) \mathbf{u}=(\operatorname{curl} \mathbf{u}) \times \mathbf{u}+\frac{1}{2} \operatorname{grad}|\mathbf{u}|^{2}
$$

that this problem can equivalently be written as, for $\varepsilon=0$ and 1 ,

$$
\begin{aligned}
& \nu \operatorname{curl}(\operatorname{curl} \mathbf{u})-\varepsilon \nu \operatorname{grad}(\operatorname{div} \mathbf{u}) \\
& +(\operatorname{curl} \mathbf{u}) \times \mathbf{u}+\operatorname{grad} p=\mathbf{f} \text { in } \Omega, \\
& \operatorname{div} \mathbf{u}=0 \quad \text { in } \Omega \text {, } \\
& \mathbf{u} \times \mathbf{n}=\mathbf{0} \quad \text { on } \Gamma \text {, } \\
& \mathbf{u} \cdot \mathbf{n}=0 \quad \text { on } \Gamma \cup \Gamma_{m}, \\
& (\operatorname{curl} \mathbf{u}) \times \mathbf{n}=\mathbf{0} \quad \text { on } \Gamma_{m},
\end{aligned}
$$

where the new unknown

$$
p=P+\frac{1}{2}|\mathbf{u}|^{2}
$$

represents the dynamic pressure. The reason for choosing this modified form is that the last boundary condition, namely $(\operatorname{curl} \mathbf{u}) \times \mathbf{n}=\mathbf{0}$ on $\Gamma_{m}$, can now be treated as a natural boundary condition. Note also that, thanks to the equation $\operatorname{div} \mathbf{u}=0$ in $\Omega$, the two formulations for $\varepsilon=0$ and $\varepsilon=1$ are equivalent in the sense of distributions; the reason for considering both of them is explained later on (see Rem. 3.3). 
We consider the full scale of Sobolev spaces $H^{s}(\Omega), s \geq 0$, provided with the usual norm $\|\cdot\|_{H^{s}(\Omega)}$ and semi-norm $|\cdot|_{H^{s}(\Omega)}$, together with their subspaces $H_{0}^{s}(\Omega)$. We introduce the domain $H(\operatorname{div}, \Omega)$ of the divergence operator, namely

$$
H(\operatorname{div}, \Omega)=\left\{\mathbf{v} \in L^{2}(\Omega)^{d} ; \operatorname{div} \mathbf{v} \in L^{2}(\Omega)\right\} .
$$

Since the normal trace operator $\mathbf{v} \mapsto \mathbf{v} \cdot \mathbf{n}$ is defined from $H(\operatorname{div}, \Omega)$ onto $H^{-\frac{1}{2}}(\partial \Omega)$, see [14], Chapter I, Theorem 2.5, we also consider its kernel

$$
H_{0}(\operatorname{div}, \Omega)=\{\mathbf{v} \in H(\operatorname{div}, \Omega) ; \mathbf{v} \cdot \mathbf{n}=0 \text { on } \partial \Omega\} .
$$

Similarly, we introduce the domain of the curl operator

$$
H(\operatorname{curl}, \Omega)=\left\{\mathbf{v} \in L^{2}(\Omega)^{3} ; \operatorname{curl} \mathbf{v} \in L^{2}(\Omega)^{3}\right\} .
$$

The tangential trace operator $\mathbf{v} \mapsto \mathbf{v} \times \mathbf{n}$ is defined on $H(\mathbf{c u r l}, \Omega)$ with values in $H^{-\frac{1}{2}}(\partial \Omega)^{3}$, see [14], Chapter I, Theorem 2.11. It can also be checked that its restriction to $\Gamma$ maps $H(\operatorname{curl}, \Omega)$ into the dual space $H_{00}^{\frac{1}{2}}(\Gamma)^{\prime}$ of $H_{00}^{\frac{1}{2}}(\Gamma)$ (see [17], Chapter I, Thm. 11.7, for the definition of the last space). So, in view of the first boundary condition in (2.1), we define the space

$$
H_{*}(\operatorname{curl}, \Omega)=\{\mathbf{v} \in H(\operatorname{curl}, \Omega) ; \mathbf{v} \times \mathbf{n}=\mathbf{0} \text { on } \Gamma\} .
$$

We set

$$
\mathbb{X}(\Omega)=H_{0}(\operatorname{div}, \Omega) \cap H_{*}(\operatorname{curl}, \Omega) .
$$

From now on, this space is equipped with the semi-norm

$$
\|\mathbf{v}\|_{\mathbb{X}(\Omega)}=\left(\|\operatorname{div} \mathbf{v}\|_{L^{2}(\Omega)}^{2}+\|\operatorname{curl} \mathbf{v}\|_{L^{2}(\Omega)^{3}}^{2}\right)^{\frac{1}{2}} .
$$

Since $\Omega$ is simply-connected, we recall from [3], Corollary 3.16, that this quantity is a norm, which is equivalent to the graph norm of $H(\operatorname{div}, \Omega) \cap H(\operatorname{curl}, \Omega)$, i.e., that there exists a constant $c$ only depending on $\Omega$ such that

$$
\forall \mathbf{v} \in \mathbb{X}(\Omega), \quad\|\mathbf{v}\|_{L^{2}(\Omega)^{3}} \leq c\|\mathbf{v}\|_{\mathbb{X}(\Omega)} .
$$

We denote by $L_{0}^{2}(\Omega)$ the space of functions in $L^{2}(\Omega)$ with a zero mean-value on $\Omega$.

We now assume that the data $\mathbf{f}$ belong to the dual space $\mathbb{X}(\Omega)^{\prime}$ of $\mathbb{X}(\Omega)$ and consider the variational problem:

Find $(\mathbf{u}, p)$ in $\mathbb{X}(\Omega) \times L_{0}^{2}(\Omega)$ such that

$$
\begin{aligned}
& \forall \mathbf{v} \in \mathbb{X}(\Omega), \quad a_{\varepsilon}(\mathbf{u}, \mathbf{v})+c(\mathbf{u}, \mathbf{u}, \mathbf{v})+b(\mathbf{v}, p)=\langle\mathbf{f}, \mathbf{v}\rangle, \\
& \forall q \in L_{0}^{2}(\Omega), \quad b(\mathbf{u}, q)=0,
\end{aligned}
$$

where the bilinear forms $a_{\varepsilon}(\cdot, \cdot)$ and $b(\cdot, \cdot)$ and the trilinear form $c(\cdot, \cdot, \cdot)$ are defined by

$$
\begin{aligned}
a_{\varepsilon}(\mathbf{u}, \mathbf{v})= & \nu \int_{\Omega}(\operatorname{curl} \mathbf{u})(\mathbf{x}) \cdot(\operatorname{curl} \mathbf{v})(\mathbf{x}) \mathrm{d} \mathbf{x} \\
& +\varepsilon \nu \int_{\Omega}(\operatorname{div} \mathbf{u})(\mathbf{x})(\operatorname{div} \mathbf{v})(\mathbf{x}) \mathrm{d} \mathbf{x}, \\
b(\mathbf{v}, q)= & -\int_{\Omega}(\operatorname{div} \mathbf{v})(\mathbf{x}) q(\mathbf{x}) \mathrm{d} \mathbf{x}, \\
c(\mathbf{w}, \mathbf{u}, \mathbf{v})= & \int_{\Omega}((\operatorname{curl} \mathbf{w})(\mathbf{x}) \times \mathbf{u}(\mathbf{x})) \cdot \mathbf{v}(\mathbf{x}) \mathrm{d} \mathbf{x} .
\end{aligned}
$$


Here, $\langle\cdot, \cdot\rangle$ stands for the duality pairing between $\mathbb{X}(\Omega)$ and its dual space. The reason for the introduction of this problem is stated in the next proposition.

Proposition 2.1. Any solution of problem (2.3) is a solution of problem (2.1) where the first two equations are satisfied in the sense of distributions.

Proof. Let $(\mathbf{u}, p)$ be a solution of problem (2.3). Denoting by $\mathcal{D}(\Omega)$ the space of infinitely differentiable functions with a compact support in $\Omega$, we first take $\mathbf{v}$ in $\mathcal{D}(\Omega)^{3}$ in the first line of problem (2.3). This gives the first equation in problem (2.1). Next, it is readily checked from the Stokes formula that the second line of problem (2.3) is also satisfied when $q$ is a constant, hence for all $q$ in $L^{2}(\Omega)$. Thus, we take $q$ in $\mathcal{D}(\Omega)$, which yields the second equation in problem (2.1). It also follows from the definition of $\mathbb{X}(\Omega)$ that the first two boundary conditions in problem (2.1) hold. Finally, introducing an infinitely differentiable function $\varphi$ with a compact support in $\Gamma_{m}$ and choosing $\mathbf{v}$ as a lifting in $\mathbb{X}(\Omega) \cap H^{1}(\Omega)^{3}$ of the extension of $\boldsymbol{\varphi} \times \mathbf{n}$ by zero to $\partial \Omega$ gives the last boundary condition of problem (2.1).

Note that the converse property, i.e., the fact that any solution of problem (2.1) is a solution of problem (2.3), would require the density of $\mathcal{D}(\Omega)^{3}$ in $\mathbb{X}(\Omega)$ which is unlikely when $\Gamma_{m}$ has a positive measure.

Next, we observe that the forms $a_{\varepsilon}(\cdot, \cdot)$ and $b(\cdot, \cdot)$ are continuous on $\mathbb{X}(\Omega) \times \mathbb{X}(\Omega)$ and $\mathbb{X}(\Omega) \times L_{0}^{2}(\Omega)$, respectively. Moreover the following ellipticity property holds for $\varepsilon=1$ :

$$
\forall \mathbf{v} \in \mathbb{X}(\Omega), \quad a_{1}(\mathbf{v}, \mathbf{v}) \geq \nu\|\mathbf{v}\|_{\mathbb{X}(\Omega)}^{2} .
$$

On the other hand, the kernel

$$
V=\left\{\mathbf{v} \in \mathbb{X}(\Omega) ; \forall q \in L_{0}^{2}(\Omega), b(\mathbf{v}, q)=0\right\}
$$

is a closed subspace of $\mathbb{X}(\Omega)$ and coincides with

$$
V=\{\mathbf{v} \in \mathbb{X}(\Omega) ; \operatorname{div} \mathbf{v}=0 \text { in } \Omega\} .
$$

This result together with the definition $(2.2)$ of the norm of $\mathbb{X}(\Omega)$ yields the following ellipticity property for $\varepsilon=0$ and 1 :

$$
\forall \mathbf{v} \in V, \quad a_{\varepsilon}(\mathbf{v}, \mathbf{v}) \geq \nu\|\mathbf{v}\|_{\mathbb{X}(\Omega)}^{2} .
$$

Finally, the inf-sup condition for $b(\cdot, \cdot)$ is a direct consequence of its analogue with $\mathbb{X}(\Omega)$ replaced by $H_{0}^{1}(\Omega)^{3}$ which can be found in [14], Chapter I, Corollary 2.4, for instance: there exists a constant $\beta>0$ such that

$$
\forall q \in L_{0}^{2}(\Omega), \quad \sup _{\mathbf{v} \in \mathbb{X}(\Omega)} \frac{b(\mathbf{v}, q)}{\|\mathbf{v}\|_{\mathbb{X}(\Omega)}} \geq \beta\|q\|_{L^{2}(\Omega)} .
$$

Combining all this with [14], Chapter I, Corollary 4.1, yields the well-posedness of the Stokes problem with the same boundary conditions as in problem (2.1).

Lemma 2.2. For any data $\mathbf{f}$ in $\mathbb{X}(\Omega)^{\prime}$, the Stokes problem

$$
\text { Find }(\mathbf{u}, p) \text { in } \mathbb{X}(\Omega) \times L_{0}^{2}(\Omega) \text { such that }
$$

$$
\begin{aligned}
& \forall \mathbf{v} \in \mathbb{X}(\Omega), \quad a_{\varepsilon}(\mathbf{u}, \mathbf{v})+b(\mathbf{v}, p)=\langle\mathbf{f}, \mathbf{v}\rangle \\
& \forall q \in L_{0}^{2}(\Omega), \quad b(\mathbf{u}, q)=0
\end{aligned}
$$

has a unique solution $(\mathbf{u}, p)$ in $\mathbb{X}(\Omega) \times L_{0}^{2}(\Omega)$. Moreover, this solution satisfies

$$
\nu\|\mathbf{u}\|_{\mathbb{X}(\Omega)}+\beta\|p\|_{L^{2}(\Omega)} \leq 3\|\mathbf{f}\|_{\mathbb{X}(\Omega)^{\prime}} .
$$


Let $\mathcal{S}$ denote the Stokes operator, i.e., the operator which associates with any $\mathbf{f}$ in $\mathbb{X}(\Omega)^{\prime}$ the part $\mathbf{u}$ of the solution $(\mathbf{u}, p)$ of problem (2.7). Lemma 2.2 states that this operator is well-defined. We now prove some further regularity properties.

Lemma 2.3. The operator $\mathcal{S}$ maps $L^{2}(\Omega)^{3}$ into $H^{s}(\Omega)^{3}$ for $s \leq \frac{1}{2}$ in the general case and $s \leq 1$ when $\Omega$ is convex.

Proof. This follows from the embedding of $H_{0}(\operatorname{div}, \Omega) \cap H(\operatorname{curl}, \Omega)$ into $H^{\frac{1}{2}}(\Omega)^{3}$ in the general case [9] and into $H^{1}(\Omega)^{3}$ when $\Omega$ is convex [3], Theorem 2.17 .

It can also be noted that, for any data $\mathbf{f}$ in $L^{2}(\Omega)^{3}$, the restrictions of $\mathcal{S} \mathbf{f}$ to any subdomain of $\Omega$ which does not intersect a neighbourhood of $\Gamma$ or a neighbourhood of $\Gamma_{m}$ is more regular. However, the singularities that appear near $\bar{\Gamma} \cap \bar{\Gamma}_{m}$ seem presently unknown. On the other hand, it is likely that, if $\Omega$ is a convex polyhedron, $\mathcal{S}$ maps $L^{2}(\Omega)^{3}$ into $H^{s}(\Omega)^{3}$ for some $s>1$. However, proving this result is beyond the scope of this work.

To study the non-linear part of the problem, we suppose that:

Assumption 2.4. The space $\mathbb{X}(\Omega)$ is compactly embedded in $L^{4}(\Omega)^{3}$.

Assumption 2.4 is always satisfied when $\Omega$ has a $\mathcal{C}^{1,1}$ boundary or is convex: indeed, it is proved in [13] or in [3], Theorem 2.17 that, in these cases, the space $H_{0}(\operatorname{div}, \Omega) \cap H(\operatorname{curl}, \Omega)$ is contained in $H^{1}(\Omega)^{3}$, hence in $L^{6}(\Omega)^{3}$. This of course yields the desired embedding. In the next lemma, we make more precise the situations where we can prove that this hypothesis is satisfied.

Lemma 2.5. Assume that $\Omega$ is a polyhedron and denote by $\mathcal{V}$ a neighbourhood of the re-entrant corners of $\Omega$ inside $\Gamma_{m}$. Then, the space of restrictions of functions of $\mathbb{X}(\Omega)$ to $\Omega \backslash \overline{\mathcal{V}}$ is compactly embedded in $L^{4}(\Omega \backslash \overline{\mathcal{V}})^{3}$.

Proof. Since this property is local, it suffices to check it for the different parts of $\Omega \backslash \overline{\mathcal{V}}$. For each such part $\Omega_{k}$, we implicitly use a regular function $\alpha_{k}$ which is equal to 1 on $\Omega_{k}$ and vanishes outside a (small enough) neighbourhood of $\Omega_{k}$ in $\Omega \backslash \overline{\mathcal{V}}$.

(1) Let $\Omega_{1}$ be a convex subset of $\Omega \backslash \overline{\mathcal{V}}$. Then, the previous arguments yield the desired property with $\Omega \backslash \overline{\mathcal{V}}$ replaced by $\Omega_{1}$.

(2) Let $\Omega_{2}$ be a subset of $\Omega \backslash \overline{\mathcal{V}}$ such that $\partial \Omega_{2} \cap \partial \Omega$ does not intersect $\bar{\Gamma}_{m}$. Since the space of functions in $H\left(\operatorname{div}, \Omega_{2}\right) \cap H\left(\mathbf{c u r l}, \Omega_{2}\right)$ with zero traces on $\partial \Omega_{2}$ coincides with $H_{0}^{1}\left(\Omega_{2}\right)^{3}$, we have the desired result on $\Omega_{2}$.

(3) Let $\Omega_{3}$ be a neighbourhood in $\Omega \backslash \overline{\mathcal{V}}$ of part of a re-entrant edge $E$ in $\bar{\Gamma}_{m}$. It follows from [10], equation $(2.1)$ that each function in $H_{0}(\operatorname{div}, \Omega) \cap H(\operatorname{curl}, \Omega)$ can be written as the sum of a function in $H^{1}\left(\Omega_{3}\right)^{3} \cap H_{0}\left(\operatorname{div}, \Omega_{3}\right)$ and of the gradient of a function $\Phi_{3}$. Moreover, this function $\Phi_{3}$ can be written as (see [10], Sect. 3.E)

where

$$
\Phi_{3}(r, \theta, z)=S(r, z) r^{\frac{\pi}{\omega}} \varphi(\theta)
$$

- $z$ stands for the tangential coordinate on $E$;

- $r$ and $\theta$ denote the polar coordinates in the dihedron with axis $E$ such that its two faces intersect $\partial \Omega_{3}$;

- $\omega$ is the angle of this dihedron;

- $S$ is a smooth cut-off function and $\varphi$ a smooth function on $[0, \omega]$ which satisfies the appropriate boundary conditions.

Thus, it is readily checked that, since $\pi<\omega<2 \pi$, the function grad $\Phi_{3}$ belongs to a compact subset of $L^{4}\left(\Omega_{3}\right)^{3}$, whence the desired embedding follows.

(4) Let $\Omega_{4}$ be a neighbourhood of the re-entrant corners or edges in $\bar{\Gamma} \cap \bar{\Gamma}_{m}$, and let $\Gamma_{4}$ denote the intersection $\Gamma \cap \partial \Omega_{4}$. Using once more [10], equation (2.1), we observe that the restriction to $\Omega_{4}$ of any function $\mathbf{v}$ in $H_{0}(\operatorname{div}, \Omega) \cap H(\mathbf{c u r l}, \Omega)$ can be written as

$$
\mathbf{v}_{\mid \Omega_{4}}=\mathbf{v}_{4}+\operatorname{grad} \Phi_{4},
$$


where $\mathbf{v}_{4}$ belongs to $H^{1}\left(\Omega_{4}\right)^{3} \cap H_{0}\left(\operatorname{div}, \Omega_{4}\right)$ and $\Phi_{4}$ is the product of a smooth cut-off function by a harmonic function $\varphi_{4}$. If, moreover, $\mathbf{v}$ belongs to $\mathbb{X}(\Omega)$, the following boundary conditions hold on $\Gamma_{4}$

$$
\mathbf{v}_{4}+\operatorname{grad} \Phi_{4}=\mathbf{0} \quad \text { on } \Gamma_{4} .
$$

By using the harmonic lifting of $\mathbf{v}_{4}$ in $H^{1}\left(\Omega_{4}\right)^{3}$, we can easily assume that $\operatorname{grad} \Phi_{4}$ vanishes on $\Gamma_{4}$. Thus, the function $\varphi_{4}$ satisfies, up to a constant,

$$
-\Delta \varphi_{4}=0 \quad \text { in } \Omega_{4}, \quad \varphi_{4}=\partial_{n} \varphi_{4}=0 \quad \text { on } \Gamma_{4} .
$$

Thus, according to Holmgren's principle (see [21], Sect. 21, for instance), it is zero, and the function $\mathbf{v}_{4}$ belongs to $H^{1}\left(\Omega_{4}\right)^{3}$.

This concludes the proof.

It follows from the previous lemma that Assumption 2.4 holds for any polyhedron without re-entrant corners inside $\Gamma_{m}$. This assumption seems necessary to prove the continuity of the form $c(\cdot, \cdot, \cdot)$.

Lemma 2.6. If Assumption 2.4 is satisfied, the form $c(\cdot, \cdot, \cdot)$ is continuous on $\mathbb{X}(\Omega) \times \mathbb{X}(\Omega) \times \mathbb{X}(\Omega)$.

Proof. Owing to Assumption 2.4, this a direct consequence of Hölder's inequality

$$
|c(\mathbf{w}, \mathbf{u}, \mathbf{v})| \leq\|\operatorname{curl} \mathbf{w}\|_{L^{2}(\Omega)^{3}}\|\mathbf{u}\|_{L^{4}(\Omega)^{3}}\|\mathbf{v}\|_{L^{4}(\Omega)^{3}} .
$$

Moreover, the following antisymmetry property is readily checked:

$$
\forall \mathbf{w} \in \mathbb{X}(\Omega), \forall \mathbf{v} \in \mathbb{X}(\Omega), \quad c(\mathbf{w}, \mathbf{v}, \mathbf{v})=0 .
$$

Thus, we are in a position to prove the existence of a solution to problem (2.3). We proceed in three steps and begin with an a priori estimate of the solution.

Proposition 2.7. If Assumption 2.4 is satisfied, for any data $\mathbf{f}$ in $\mathbb{X}(\Omega)^{\prime}$, any solution $(\mathbf{u}, p)$ of problem (2.3) satisfies

$$
\begin{aligned}
\|\mathbf{u}\|_{\mathbb{X}(\Omega)} & \leq \frac{1}{\nu}\|\mathbf{f}\|_{\mathbb{X}(\Omega)^{\prime}} \\
\|p\|_{L^{2}(\Omega)} & \leq c\|\mathbf{f}\|_{\mathbb{X}(\Omega)^{\prime}}\left(1+\|\mathbf{f}\|_{\mathbb{X}(\Omega)^{\prime}}\right) .
\end{aligned}
$$

Proof. When taking v equal to $\mathbf{u}$ in problem (2.3) and using (2.5) and property (2.9), we obtain the estimate for $\mathbf{u}$ in (2.10) with the desired constant. The estimate for $p$ is then easily derived from the inf-sup condition (2.6).

Proposition 2.8. If Assumption 2.4 is satisfied, there exists a real number $\nu_{0}>0$ such that, for $\nu \geq \nu_{0}$ and for any data $\mathbf{f}$ in $\mathbb{X}(\Omega)^{\prime}$, problem (2.3) has a unique solution $(\mathbf{u}, p)$.

Proof. With any function $\mathbf{u}$ in $\mathbb{X}(\Omega)$, we associate the function

$$
\Phi(\mathbf{u})=\mathcal{S}(\mathbf{f}-(\operatorname{curl} \mathbf{u}) \times \mathbf{u}) .
$$

(1) Taking $\mathbf{v}$ equal to $\Phi(\mathbf{u})$ in the equation defining $\Phi(\mathbf{u})$, we derive from inequality (2.5) and Assumption 2.4 that

$$
\|\Phi(\mathbf{u})\|_{\mathbb{X}(\Omega)} \leq \frac{1}{\nu}\|\mathbf{f}\|_{\mathbb{X}(\Omega)^{\prime}}+\frac{c_{0}^{2}}{\nu}\|\mathbf{u}\|_{\mathbb{X}(\Omega)}^{2}
$$


where $c_{0}$ stands for the norm of the embedding of $\mathbb{X}(\Omega)$ into $L^{4}(\Omega)^{3}$. We now set:

$$
\begin{aligned}
& \nu_{0}>2 c_{0} \sqrt{\|\mathbf{f}\|_{\mathbb{X}(\Omega)^{\prime}}}, \\
& R=\frac{\nu}{2 c_{0}^{2}}\left(1-\sqrt{1-\frac{4 c_{0}^{2}}{\nu^{2}}\|\mathbf{f}\|_{\mathbb{X}(\Omega)^{\prime}}}\right) .
\end{aligned}
$$

It thus follows from the previous lines that, for $\nu \geq \nu_{0}, \Phi$ maps the ball with radius $R$ into itself.

(2) Let $\mathbf{u}_{1}$ and $\mathbf{u}_{2}$ be two functions in the ball with radius $R$. The same arguments previously used yield that

$$
\begin{aligned}
\left\|\Phi\left(\mathbf{u}_{1}\right)-\Phi\left(\mathbf{u}_{2}\right)\right\|_{\mathbb{X}(\Omega)} & \leq \frac{1}{\nu} \sup _{\mathbf{v} \in \mathbb{X}(\Omega)} \frac{c\left(\mathbf{u}_{1}-\mathbf{u}_{2}, \mathbf{u}_{1}, \mathbf{v}\right)+c\left(\mathbf{u}_{2}, \mathbf{u}_{1}-\mathbf{u}_{2}, \mathbf{v}\right)}{\|\mathbf{v}\|_{\mathbb{X}(\Omega)}} \\
& \leq \frac{2 c_{0}^{2} R}{\nu}\left\|\mathbf{u}_{1}-\mathbf{u}_{2}\right\|_{\mathbb{X}(\Omega)}
\end{aligned}
$$

When $\nu \geq \nu_{0}$ the choice of $R$ implies that the quantity $\frac{2 c_{0}^{2} R}{\nu}$ is less than 1 . Hence, the mapping $\Phi$ is a contraction of the ball with radius $R$.

(3) Combining the first two parts of the proof with Banach's fixed point theorem yields the existence of a function $\mathbf{u}$ in $\mathbb{X}(\Omega)$ such that $\Phi(\mathbf{u})=\mathbf{u}$. Denoting by $p$ the function associated with this $\Phi(\mathbf{u})$ in problem (2.7), we observe that the pair $(\mathbf{u}, p)$ is a solution of problem $(2.3)$.

(4) Let $\left(\mathbf{u}_{1}, p_{1}\right)$ and $\left(\mathbf{u}_{2}, p_{2}\right)$ be two solutions of problem (2.3). The same arguments as in part (2) of the proof combined with the estimates for $\mathbf{u}_{1}$ and $\mathbf{u}_{2}$ established in Proposition 2.7 lead to

$$
\left\|\mathbf{u}_{1}-\mathbf{u}_{2}\right\|_{\mathbb{X}(\Omega)} \leq \frac{2 c_{0}^{2}}{\nu^{2}}\|\mathbf{f}\|_{\mathbb{X}(\Omega)^{\prime}}\left\|\mathbf{u}_{1}-\mathbf{u}_{2}\right\|_{\mathbb{X}(\Omega)}
$$

When $\nu \geq \nu_{0}$, this inequality implies that $\mathbf{u}_{1}$ is equal to $\mathbf{u}_{2}$. Next, by applying the inf-sup condition (2.6), it is readily checked that $p_{1}$ and $p_{2}$ coincide. This gives the uniqueness of the solution.

Proposition 2.9. If Assumption 2.4 is satisfied, for all $\nu>0$ and for any data $\mathbf{f}$ in $\mathbb{X}(\Omega)^{\prime}$, problem (2.3) has at least a solution $(\mathbf{u}, p)$.

Proof. Since the result for $\nu \geq \nu_{0}$ is proven in Proposition 2.8, we assume that $0<\nu<\nu_{0}$. Denoting by $\mathcal{S}_{*}$ the Stokes operator $\mathcal{S}$ for the viscosity $\nu$ equal to 1 and setting $\mu=\frac{1}{\nu}$, we observe that problem (2.3) can equivalently be written (up to a modification of the pressure) as

$$
\mathbf{u}+\mu \mathcal{S}_{*}((\operatorname{curl} \mathbf{u}) \times \mathbf{u}-\mathbf{f}) .
$$

We now intend to prove the existence of a solution for this problem, for $\mu$ in the interval $\left[\mu_{0}, \mu_{1}\right]$ with $\mu_{0}=\frac{1}{\nu_{0}}$, $\mu_{1}=\frac{1}{\nu}$ by using the Leray-Schauder theory (see [16], Chapter 2, Sect. 5, for instance).

(1) Let $B$ denote the open ball of $\mathbb{X}(\Omega)$ with radius $2 \mu_{1}\|\mathbf{f}\|_{\mathbb{X}(\Omega)^{\prime}}$. It follows from Proposition 2.7 that, for any $\mu \in\left[\mu_{0}, \mu_{1}\right]$, equation $(2.11)$ has no solution $\mathbf{u}$ such that $\|\mathbf{u}\|_{\mathbb{X}(\Omega)}=2 \mu_{1}\|\mathbf{f}\|_{\mathbb{X}(\Omega)^{\prime}}$.

(2) Let $\Psi$ be the mapping $(\mu, \mathbf{v}) \mapsto \mathbf{v}+\mu \mathcal{S}_{*}((\operatorname{curl} \mathbf{v}) \times \mathbf{v}-\mathbf{f})$. The mapping $\Psi$ is continuously differentiable on $\left[\mu_{0}, \mu_{1}\right] \times \mathbb{X}(\Omega)$, and it follows from Assumption 2.4, more precisely from the compactness of the embedding of $\mathbb{X}(\Omega)$ into $L^{4}(\Omega)^{3}$, that the mapping Id $-\Psi$ maps $\left[\mu_{0}, \mu_{1}\right] \times \bar{B}$ into a compact subset of $\mathbb{X}(\Omega)$.

(3) Proposition 2.8 yields that, for $\mu=\mu_{0}$, equation (2.11) has a unique solution $\mathbf{u}$. Then, this solution is nonsingular in the sense that the Fréchet derivative of $\Psi$ with respect to $\mathbf{v}$ at $\left(\mu_{0}, \mathbf{u}\right)$ is an isomorphism of $\mathbb{X}(\Omega)$. Thus the topological degree of $\Psi\left(\mu_{0}, \cdot\right)$ with respect to $B$ is not zero (we skip the technical argument which is needed to check this result and which relies on the fact that the compact operator $D(\mathrm{Id}-\Psi)$ is the limit of operators with finite-dimensional range). 
(4) Since the topological degree is invariant under homotopy, the degree of $\Psi\left(\mu_{1}, \cdot\right)$ with respect to $B$ is not zero. This yields the existence of a solution $\mathbf{u}$ of equation (2.11) for $\mu=\mu_{1}$.

(5) Using the inf-sup condition (2.6), we easily derive the existence of a $p$ in $L_{0}^{2}(\Omega)$ such that $(\mathbf{u}, p)$ is a solution of problem (2.3).

Remark 2.10. By combining Lemma 2.3 with a boot-strap argument, we easily derive that any solution (u, $p$ ) of problem (2.3) satisfies the same regularity properties as the solution of problem (2.7). For instance, for any data $\mathbf{f}$ in $L^{2}(\Omega)^{3}$, the velocity $\mathbf{u}$ belongs to $H^{1}(\Omega)^{3}$ when $\Omega$ is convex.

To conclude, we note that all results of this section hold for both $\varepsilon=0$ and $\varepsilon=1$. Working with $\varepsilon=0$ seems simpler. But, as we will see in the next section, the formulation with $\varepsilon=1$ is advantageous for the discretization. Therefore we work from now on with $\varepsilon=1$. For simplicity, we write $a(\cdot, \cdot)$ instead of $a_{1}(\cdot, \cdot)$ in what follows.

\section{The DisCRETE PROBLEM}

From now on, we assume that $\Omega$ is a polyhedron. We introduce a regular family of triangulations $\left(\mathcal{T}_{h}\right)_{h}$ by tetrahedra, in the usual sense that:

- For each $h, \bar{\Omega}$ is the union of all elements of $\mathcal{T}_{h}$;

- The intersection of two different elements of $\mathcal{T}_{h}$, if not empty, is a vertex or a whole edge or a whole face of both of them;

- The ratio of the diameter $h_{K}$ of any element $K$ of $\mathcal{T}_{h}$ to the diameter of its inscribed sphere is smaller than a constant $\sigma$ independent of $h$.

Further we make the non restrictive assumption that $\bar{\Gamma}_{m}$ is the union of whole faces of elements of $\mathcal{T}_{h}$.

As usual, $h$ denotes the maximum of the diameters $h_{K}, K \in \mathcal{T}_{h}$. For each $K$ in $\mathcal{T}_{h}$ and each nonnegative integer $k$, we denote by $\mathcal{P}_{k}(K)$ the spaces of restrictions to $K$ of polynomials with three variables and total degree at most $k$.

In what follows, $c, c^{\prime} \ldots$ stand for generic constants which may vary from line to line but are always independent of $h$. From now on, we call finite element space associated with $\mathcal{T}_{h}$ a space of functions such that their restrictions to any element $K$ of $\mathcal{T}_{h}$ belong to a space of polynomials of fixed degree.

For each $h$, we associate with $\mathcal{T}_{h}$ two finite element spaces $\mathbb{X}_{h}$ and $\mathbb{M}_{h}$ which are contained in $\mathbb{X}(\Omega)$ and $L_{0}^{2}(\Omega)$, respectively, and such that the following inf-sup condition holds for a constant $\beta_{*}>0$ :

$$
\forall q_{h} \in \mathbb{M}_{h}, \quad \sup _{\mathbf{v}_{h} \in \mathbb{X}_{h}} \frac{b\left(\mathbf{v}_{h}, q_{h}\right)}{\left\|\mathbf{v}_{h}\right\|_{\mathbb{X}(\Omega)}} \geq \beta_{*}\left\|q_{h}\right\|_{L^{2}(\Omega)}
$$

Indeed, there exist many examples of finite element spaces satisfying these conditions (the inf-sup condition being usually proved with $\mathbb{X}_{h}$ replaced by $\left.\mathbb{X}_{h} \cap H_{0}^{1}(\Omega)^{3}\right)$, see [14], Chapter II. We give two examples of them, the second one dealing with continuous discrete pressures.

Example 3.1. Spaces associated with the Bernardi-Raugel elements (see e.g. [14], Sect. II.2.1)

$$
\mathbb{X}_{h}=\left\{\mathbf{v}_{h} \in \mathbb{X}(\Omega) ; \forall K \in \mathcal{T}_{h}, \mathbf{v}_{h \mid K} \in \mathcal{P}(K)\right\}
$$

where $\mathcal{P}(K)$ stands for the space spanned by the restrictions to $K$ of functions in $\mathcal{P}_{1}(K)^{3}$ and the normal bubble functions $\psi_{e} \mathbf{n}_{e}$ (for each face $e$ of $K, \psi_{e}$ denotes the bubble function on $e$ equal to the product of the barycentric coordinates associated with the vertices of $e$ and $\mathbf{n}_{e}$ stands for the unit outward normal vector on $e$ ),

$$
\mathbb{M}_{h}=\left\{q_{h} \in L_{0}^{2}(\Omega) ; \forall K \in \mathcal{T}_{h}, q_{h \mid K} \in \mathcal{P}_{0}(K)\right\}
$$


Example 3.2. Spaces associated with the Taylor-Hood elements (see e.g. [14], Sect. II.4.2)

$$
\begin{gathered}
\mathbb{X}_{h}=\left\{\mathbf{v}_{h} \in \mathbb{X}(\Omega) ; \forall K \in \mathcal{T}_{h}, \mathbf{v}_{h \mid K} \in \mathcal{P}_{2}(K)^{3}\right\} \\
\mathbb{M}_{h}=\left\{q_{h} \in L_{0}^{2}(\Omega) \cap H^{1}(\Omega) ; \forall K \in \mathcal{T}_{h}, q_{h \mid K} \in \mathcal{P}_{1}(K)\right\} .
\end{gathered}
$$

The discrete problem is obtained from problem $(2.3)$ (with $\varepsilon=1$ ) by the Galerkin method. It reads:

Find $\left(\mathbf{u}_{h}, p_{h}\right)$ in $\mathbb{X}_{h} \times \mathbb{M}_{h}$ such that

$$
\begin{aligned}
& \forall \mathbf{v}_{h} \in \mathbb{X}_{h}, \quad a\left(\mathbf{u}_{h}, \mathbf{v}_{h}\right)+c\left(\mathbf{u}_{h}, \mathbf{u}_{h}, \mathbf{v}_{h}\right)+b\left(\mathbf{v}_{h}, p_{h}\right)=\left\langle\mathbf{f}, \mathbf{v}_{h}\right\rangle, \\
& \forall q_{h} \in \mathbb{M}_{h}, \quad b\left(\mathbf{u}_{h}, q_{h}\right)=0 .
\end{aligned}
$$

It follows from the choice of $\mathbb{X}_{h}$ and $\mathbb{M}_{h}$ that the discretization is fully conforming. As a consequence, the forms $a(\cdot, \cdot), b(\cdot, \cdot)$ and - if Assumption 2.4 holds $-c(\cdot, \cdot, \cdot)$ are continuous on $\mathbb{X}_{h} \times \mathbb{X}_{h}, \mathbb{X}_{h} \times \mathbb{M}_{h}$ and $\mathbb{X}_{h} \times \mathbb{X}_{h} \times \mathbb{X}_{h}$, respectively, with norms bounded independently of $h$. Moreover, an immediate consequence of the ellipticity property (2.4) is its discrete analogue

$$
\forall \mathbf{v}_{h} \in \mathbb{X}_{h}, \quad a\left(\mathbf{v}_{h}, \mathbf{v}_{h}\right) \geq \nu\left\|\mathbf{v}_{h}\right\|_{\mathbb{X}(\Omega)}^{2}
$$

Remark 3.3. As usual, we denote by $V_{h}$ the kernel

$$
V_{h}=\left\{\mathbf{v}_{h} \in \mathbb{X}_{h} ; \forall q_{h} \in \mathbb{M}_{h}, b\left(\mathbf{v}_{h}, q_{h}\right)=0\right\}
$$

We have no examples of finite elements such that the space $V_{h}$ consists of exactly divergence-free functions, i.e., it coincides with $\mathbb{X}_{h} \cap V$. Indeed, this seems in contradiction with the inf-sup condition (3.1) (see [14], Chapter II, or [6], Chapter VI, for more comments). For this reason, the discrete analogue of the ellipticity property (2.5) does not hold, and we are led to work with $\varepsilon=1$.

The antisymmetry property (2.9) also holds for all functions $\mathbf{w}_{h}$ and $\mathbf{v}_{h}$ in $\mathbb{X}_{h}$. So using the same arguments as in the proof of Proposition 2.7 combined with inequalities (3.1) and (3.3), we obtain an a priori estimate for the solutions of problem (3.2).

Proposition 3.4. For any data $\mathbf{f}$ in $\mathbb{X}(\Omega)^{\prime}$, any solution $\left(\mathbf{u}_{h}, p_{h}\right)$ of problem (3.2) satisfies

$$
\begin{aligned}
\left\|\mathbf{u}_{h}\right\|_{\mathbb{X}(\Omega)} & \leq \frac{1}{\nu}\|\mathbf{f}\|_{\mathbb{X}(\Omega)^{\prime}} \\
\left\|p_{h}\right\|_{L^{2}(\Omega)} & \leq c\|\mathbf{f}\|_{\mathbb{X}(\Omega)^{\prime}}\left(1+\|\mathbf{f}\|_{\mathbb{X}(\Omega)^{\prime}}\right) .
\end{aligned}
$$

\section{Existence of A SOlution And A PRIORI ERror ESTimates}

Our approach for proving the existence of a solution to problem (3.2) relies on the theory of Brezzi et al. [7]. So we first investigate the discretization of the linear problem (2.7).

For this, we need a further assumption concerning the approximation properties of the spaces $\mathbb{X}_{h}$ and $\mathbb{M}_{h}$.

Assumption 4.1. There exists an integer $k \geq 1$ such that the following approximation properties hold for any function $\mathbf{v}$ in $\mathbb{X}(\Omega) \cap H^{s}(\Omega)^{3}$ and $q$ in $L_{0}^{2}(\Omega) \cap H^{s-1}(\Omega), 1 \leq s \leq k+1$,

$$
\begin{gathered}
\inf _{\mathbf{v}_{h} \in \mathbb{X}_{h}}\left\|\mathbf{v}-\mathbf{v}_{h}\right\|_{\mathbb{X}(\Omega)} \leq c h^{s-1}\|\mathbf{v}\|_{H^{s}(\Omega)^{3}}, \\
\inf _{q \in \mathbb{M}_{h}}\left\|q-q_{h}\right\|_{L^{2}(\Omega)} \leq c h^{s-1}\|q\|_{H^{s-1}(\Omega)} .
\end{gathered}
$$

It can be observed that this assumption holds with $k=1$ for Example 3.1 and with $k=2$ for Example 3.2 .

For any data $\mathbf{f}$ in $\mathbb{X}(\Omega)^{\prime}$, we consider the discrete Stokes problem: 
Find $\left(\mathbf{u}_{h}, p_{h}\right)$ in $\mathbb{X}_{h} \times \mathbb{M}_{h}$ such that

$$
\begin{aligned}
& \forall \mathbf{v}_{h} \in \mathbb{X}_{h}, \quad a\left(\mathbf{u}_{h}, \mathbf{v}_{h}\right)+b\left(\mathbf{v}_{h}, p_{h}\right)=\left\langle\mathbf{f}, \mathbf{v}_{h}\right\rangle, \\
& \forall q_{h} \in \mathbb{M}_{h}, \quad b\left(\mathbf{u}_{h}, q_{h}\right)=0 .
\end{aligned}
$$

It follows from inequalities (3.1) and (3.3) that this problem has a unique solution.

We denote by $\mathcal{S}_{h}$ the operator which associates with any $\mathbf{f}$ in $\mathbb{X}(\Omega)^{\prime}$ the part $\mathbf{u}_{h}$ of the solution $\left(\mathbf{u}_{h}, p_{h}\right)$ of problem (4.2). The first property of $\mathcal{S}_{h}$ is easily derived from inequality (3.3).

Lemma 4.2. The following stability property holds for any $\mathbf{f}$ in $\mathbb{X}(\Omega)^{\prime}$ :

$$
\left\|\mathcal{S}_{h} \mathbf{f}\right\|_{\mathbb{X}(\Omega)} \leq \frac{1}{\nu}\|\mathbf{f}\|_{\mathbb{X}(\Omega)^{\prime}}
$$

We also need an a priori error estimate between the solutions $\mathbf{u}$ of problem (2.7) and $\mathbf{u}_{h}$ of problem (4.2).

Lemma 4.3. If Assumption 4.1 holds, for any $\mathbf{f}$ in $\mathbb{X}(\Omega)^{\prime} \cap H^{s-2}(\Omega)^{3}$ such that $\mathcal{S} \mathbf{f}$ belongs to $H^{s}(\Omega)^{3}$ with $1 \leq s \leq k+1$, the error estimate

$$
\left\|\left(\mathcal{S}-\mathcal{S}_{h}\right) \mathbf{f}\right\|_{\mathbb{X}(\Omega)} \leq c h^{s-1}\left(\|\mathcal{S} \mathbf{f}\|_{H^{s}(\Omega)^{3}}+\|\mathbf{f}\|_{H^{s-2}(\Omega)^{3}}\right)
$$

is fulfilled.

Proof. Let $p$ be the pressure associated with $\mathbf{u}=\mathcal{S} \mathbf{f}$ in problem (2.7). The functions $\mathbf{u}$ and $\mathbf{u}_{h}=\mathcal{S}_{h} \mathbf{f}$ satisfy

$$
\begin{aligned}
\forall \mathbf{v}_{h} \in V_{h}, & \forall q_{h} \in \mathbb{M}_{h}, \quad a\left(\mathbf{u}, \mathbf{v}_{h}\right)=\left\langle\mathbf{f}, \mathbf{v}_{h}\right\rangle-b\left(\mathbf{v}_{h}, p-q_{h}\right), \\
\forall \mathbf{v}_{h} \in V_{h}, & a\left(\mathbf{u}_{h}, \mathbf{v}_{h}\right)=\left\langle\mathbf{f}, \mathbf{v}_{h}\right\rangle .
\end{aligned}
$$

It thus follows from the ellipticity property (3.3) that

$$
\left\|\mathbf{u}-\mathbf{u}_{h}\right\|_{\mathbb{X}(\Omega)} \leq c\left(\inf _{\mathbf{w}_{h} \in V_{h}}\left\|\mathbf{u}-\mathbf{w}_{h}\right\|_{\mathbb{X}(\Omega)}+\inf _{q \in \mathbb{M}_{h}}\left\|p-q_{h}\right\|_{L^{2}(\Omega)}\right) .
$$

Next, combining the inf-sup condition (3.1) with [14], Chapter II, equation (1.16) gives

$$
\left\|\mathbf{u}-\mathbf{u}_{h}\right\|_{\mathbb{X}(\Omega)} \leq c\left(\inf _{\mathbf{w}_{h} \in \mathbb{X}_{h}}\left\|\mathbf{u}-\mathbf{w}_{h}\right\|_{\mathbb{X}(\Omega)}+\inf _{q \in \mathbb{M}_{h}}\left\|p-q_{h}\right\|_{L^{2}(\Omega)}\right) .
$$

Finally, it follows from the regularity assumptions on $\mathbf{f}$ and $\mathbf{u}$ that $p$ belongs to $H^{s-1}(\Omega)$ and satisfies

$$
\|p\|_{H^{s-1}(\Omega)} \leq c\left(\|\mathcal{S} \mathbf{f}\|_{H^{s}(\Omega)^{3}}+\|\mathbf{f}\|_{H^{s-2}(\Omega)^{3}}\right) .
$$

Thus, the desired estimate follows from the approximation properties stated in Assumption 4.1.

We now set

$$
\mathcal{G}(\mathbf{u})=(\operatorname{curl} \mathbf{u}) \times \mathbf{u}-\mathbf{f},
$$

and observe that problem (2.3) can equivalently be written as

$$
\mathbf{u}+\mathcal{S G}(\mathbf{u})=\mathbf{0}
$$

Similarly, problem (3.2) can equivalently be written as

$$
\mathbf{u}_{h}+\mathcal{S}_{h} \mathcal{G}\left(\mathbf{u}_{h}\right)=\mathbf{0}
$$

This new formulation is needed to apply the Brezzi-Rappaz-Raviart theorem. We now consider a solution of problem (2.3). Denoting by $D$ the Fréchet derivative assume that: 
Assumption 4.4. The solution $(\mathbf{u}, p)$ of problem (2.3) is such that

(1) it belongs to $H^{s}(\Omega)^{3} \times H^{s-1}(\Omega)$ and curl $\mathbf{u}$ belongs to $H^{s-\frac{3}{4}}(\Omega)^{3}$ for some $s>\frac{3}{2}$;

(2) the operator $\operatorname{Id}+\mathcal{S} D \mathcal{G}(\mathbf{u})$ is an isomorphism of $\mathbb{X}(\Omega)$.

Part (1) of this assumption states a regularity result for the solution $(\mathbf{u}, p)$ of our problem. Even if the regularity results for the Stokes problem with mixed boundary conditions in a three-dimensional domain are presently unknown, this property seems likely for a convex polyhedron (see [18] for the first results in dimension 2) and can easily be extended to the nonlinear case. On the other hand, part (2) means that the solution $\mathbf{u}$ is locally unique, which is much weaker than the global uniqueness, see Proposition 2.8.

In view of the next lemma, we also make a further assumption. It is likely satisfied when $\Omega$ is convex; however, we think that it is weaker.

Assumption 4.5. The operator $\mathcal{S}$ maps $L^{2}(\Omega)^{3}$ into $H^{t}(\Omega)^{3}$ for some $t>1$.

Lemma 4.6. If Assumptions 2.4, 4.1, 4.4 and 4.5 are satisfied, there exists a real number $h_{0}>0$ such that, for all $h \leq h_{0}$, the operator $\operatorname{Id}+\mathcal{S}_{h} D \mathcal{G}(\mathbf{u})$ is an isomorphism of $\mathbb{X}(\Omega)$. Moreover, the norm of its inverse is bounded independently of $h$.

Proof. We take advantage of the expansion

$$
\operatorname{Id}+\mathcal{S}_{h} D \mathcal{G}(\mathbf{u})=\operatorname{Id}+\mathcal{S} D \mathcal{G}(\mathbf{u})-\left(\mathcal{S}-\mathcal{S}_{h}\right) D \mathcal{G}(\mathbf{u})
$$

Therefore, owing to Part (2) of Assumption 4.4, it suffices to check that $\left(\mathcal{S}-\mathcal{S}_{h}\right) D \mathcal{G}(\mathbf{u})$ tends to zero in the norm of endomorphisms of $\mathbb{X}(\Omega)$. To prove this, we observe that for any $\mathbf{z}$ in $\mathbb{X}(\Omega)$,

$$
D \mathcal{G}(\mathbf{u}) \cdot \mathbf{z}=(\operatorname{curl} \mathbf{u}) \times \mathbf{z}+(\operatorname{curl} \mathbf{z}) \times \mathbf{u} .
$$

Using Assumptions 2.4 and 4.4 and noting that the embeddings of $H^{s}(\Omega)$ and $H^{s-\frac{3}{4}}(\Omega)$ into $L^{\infty}(\Omega)$ and $L^{4}(\Omega)$, respectively, are compact, we conclude that the mapping $\mathbf{z} \mapsto D \mathcal{G}(\mathbf{u}) \cdot \mathbf{z}$ maps the unit sphere of $\mathbb{X}(\Omega)$ into a compact subset of $L^{2}(\Omega)^{3}$. Owing to Assumption 4.5, the operator $\mathcal{S}$ maps this compact set into a compact subset of $H^{t}(\Omega)^{3}$. The desired convergence property then is a direct consequence of Lemma 4.3.

From now on we denote by $\mathcal{L}(\mathbb{X}(\Omega))$ the space of endomorphisms of $\mathbb{X}(\Omega)$.

Lemma 4.7. If Assumption 2.4 is satisfied, there exists a real number $L>0$ such that the following Lipschitz property holds

$$
\forall \mathbf{w} \in \mathbb{X}(\Omega), \quad\left\|\mathcal{S}_{h}(D \mathcal{G}(\mathbf{u})-D \mathcal{G}(\mathbf{w}))\right\|_{\mathcal{L}(\mathbb{X}(\Omega))} \leq L\|\mathbf{u}-\mathbf{w}\|_{\mathbb{X}(\Omega)}
$$

Proof. We now write

$$
D \mathcal{G}(\mathbf{u}) \cdot \mathbf{z}-D \mathcal{G}(\mathbf{w}) \cdot \mathbf{z}=(\operatorname{curl}(\mathbf{u}-\mathbf{w})) \times \mathbf{z}+(\operatorname{curl} \mathbf{z}) \times(\mathbf{u}-\mathbf{w}) .
$$

Owing to Assumption 2.4, this gives

$$
\|D \mathcal{G}(\mathbf{u}) \cdot \mathbf{z}-D \mathcal{G}(\mathbf{w}) \cdot \mathbf{z}\|_{\mathbb{X}(\Omega)^{\prime}} \leq c_{0}^{2}\|\mathbf{u}-\mathbf{w}\|_{\mathbb{X}(\Omega)}\|\mathbf{z}\|_{\mathbb{X}(\Omega)}
$$

Combining this with Lemma 4.2 yields the desired property.

Lemma 4.8. If Assumptions 4.1 and 4.4 are satisfied, the following estimate holds

$$
\left\|\mathbf{u}+\mathcal{S}_{h} \mathcal{G}(\mathbf{u})\right\|_{\mathbb{X}(\Omega)} \leq c h^{s^{*}-1}\left(\|\mathbf{u}\|_{H^{s}(\Omega)^{3}}+\|\mathbf{f}\|_{H^{s-2}(\Omega)^{3}}\right),
$$

with $s^{*}=\min \{s, k+1\}$. 
Proof. We have

$$
\left\|\mathbf{u}+\mathcal{S}_{h} \mathcal{G}(\mathbf{u})\right\|_{\mathbb{X}(\Omega)}=\left\|\left(\mathcal{S}-\mathcal{S}_{h}\right) \mathcal{G}(\mathbf{u})\right\|_{\mathbb{X}(\Omega)} .
$$

Estimate (4.5) then is a direct consequence of equation (4.3), Assumption 4.4 and Lemma 4.3.

We now state and prove the main result of this section.

Proposition 4.9. Suppose that Assumptions 2.4, 4.1 and 4.5 hold and that the solution (u,p) satisfies Assumption 4.4. Then, there exists a neighbourhood $\mathcal{U}$ of $\mathbf{u}$ in $\mathbb{X}(\Omega)$ such that problem (3.2) has a unique solution $\left(\mathbf{u}_{h}, p_{h}\right)$ with $\mathbf{u}_{h}$ in $\mathcal{U}$. Moreover, this solution satisfies the following a priori error estimate

$$
\left\|\mathbf{u}-\mathbf{u}_{h}\right\|_{\mathbb{X}(\Omega)}+\left\|p-p_{h}\right\|_{L^{2}(\Omega)} \leq c h^{s^{*}-1}\left(\|\mathbf{u}\|_{H^{s}(\Omega)^{3}}+\|p\|_{H^{s-1}(\Omega)}\right),
$$

with $s^{*}=\min \{s, k+1\}$.

Proof. By combining the Brezzi-Rappaz-Raviart theorem [7] (see also [14], Chapter IV, Thm. 3.1) with Lemmas 4.6 to 4.8, we derive the existence of a neighbourhood $\mathcal{U}$ of $\mathbf{u}$ such that problem (4.4) has a unique solution $\mathbf{u}_{h}$ in $\mathcal{U}$, together with the estimate for $\left\|\mathbf{u}-\mathbf{u}_{h}\right\|_{\mathbb{X}(\Omega)}$. On the other hand, it follows from the inf-sup condition (3.1) that there exists a unique $p_{h}$ in $\mathbb{M}_{h}$ such that

$$
\forall \mathbf{v}_{h} \in \mathbb{X}_{h}, \quad b\left(\mathbf{v}_{h}, p_{h}\right)=\left\langle\mathbf{f}, \mathbf{v}_{h}\right\rangle-a\left(\mathbf{u}_{h}, \mathbf{v}_{h}\right)-c\left(\mathbf{u}_{h}, \mathbf{u}_{h}, \mathbf{v}_{h}\right) .
$$

Thus, the pair $\left(\mathbf{u}_{h}, p_{h}\right)$ is a solution of problem (3.2). Moreover, by subtracting problem (3.2) from problem (2.3) and noting that the norms of both $\mathbf{u}$ and $\mathbf{u}_{h}$ are bounded independently of $h$ (see Props. 2.7 and 3.4), we obtain

$$
\left\|p-p_{h}\right\|_{L^{2}(\Omega)} \leq c\left(\left\|\mathbf{u}-\mathbf{u}_{h}\right\|_{\mathbb{X}(\Omega)}+\inf _{q_{h} \in \mathbb{M}_{h}}\left\|p-q_{h}\right\|_{L^{2}(\Omega)}\right) .
$$

Thus, the estimate for $\left\|p-p_{h}\right\|_{L^{2}(\Omega)}$ follows from the estimate for $\left\|\mathbf{u}-\mathbf{u}_{h}\right\|_{\mathbb{X}(\Omega)}$ and the approximation properties of the space $\mathbb{M}_{h}$.

Estimate (4.6) is fully optimal, and the regularity which is required for $(\mathbf{u}, p)$ seems reasonable. However, Assumptions 2.4 and 4.5 apparently add limitations to the geometry of the domain.

\section{A POSTERIORI ERROR ESTIMATES}

We first recall some standard notation: for each $K$ in $\mathcal{T}_{h}, \mathcal{E}_{K}$ denotes the set of all faces of $K$ which are not contained in $\partial \Omega$ and $\mathcal{E}_{K}^{m}$ the set of faces of $K$ which are contained in $\bar{\Gamma}_{m}$. Next, we introduce an approximation $\mathbf{f}_{h}$ of the data $\mathbf{f}$ which is constant on each element of $\mathcal{T}_{h}$.

We are thus in a position to define the error indicators. However, as in Section 2, we propose two families associated with the values 0 and 1 of the parameter $\varepsilon$. For $\varepsilon$ equal to 0 and 1 and for each $K$ in $\mathcal{T}_{h}$, the indicator $\eta_{K}^{\varepsilon}$ is given by

$$
\begin{aligned}
\eta_{K}^{\varepsilon}= & h_{K}\left\|\mathbf{f}_{h}-\nu \operatorname{curl}\left(\operatorname{curl} \mathbf{u}_{h}\right)+\varepsilon \nu \operatorname{grad}\left(\operatorname{div} \mathbf{u}_{h}\right)-\left(\operatorname{curl} \mathbf{u}_{h}\right) \times \mathbf{u}_{h}-\operatorname{grad} p_{h}\right\|_{L^{2}(K)^{3}} \\
& +\sum_{e \in \mathcal{E}_{K}} h_{e}^{\frac{1}{2}}\left\|\left[\nu\left(\operatorname{curl} \mathbf{u}_{h}\right) \times \mathbf{n}+\varepsilon \nu\left(\operatorname{div} \mathbf{u}_{h}\right) \mathbf{n}-p_{h} \mathbf{n}\right]_{e}\right\|_{L^{2}(e)^{3}} \\
& +\sum_{e \in \mathcal{E}_{K}^{m}} h_{e}^{\frac{1}{2}}\left\|\nu\left(\operatorname{curl} \mathbf{u}_{h}\right) \times \mathbf{n}\right\|_{L^{2}(e)^{3}}+\left\|\operatorname{div} \mathbf{u}_{h}\right\|_{L^{2}(K)},
\end{aligned}
$$

where

- for each $e$ in $\mathcal{E}_{K}$ or $\mathcal{E}_{K}^{m}, h_{e}$ stands for the diameter of $e$;

- for each $e$ in $\mathcal{E}_{K},[\cdot]_{e}$ denotes the jump across $e$ in the direction of $\mathbf{n}$ which is a unit vector orthogonal to $e$. 
In view of the discrete problem (3.2), it seems more natural to use the indicators $\eta_{K}^{1}$. However, triangle inequalities combined with the standard inverse inequality (see [5], Sect. XI.2, for instance)

$$
h_{K}\left\|\nu \operatorname{grad}\left(\operatorname{div} \mathbf{u}_{h}\right)\right\|_{L^{2}(K)^{3}}+\sum_{e \in \mathcal{E}_{K}} h_{e}^{\frac{1}{2}}\left\|\left[\nu\left(\operatorname{div} \mathbf{u}_{h}\right) \mathbf{n}\right]_{e}\right\|_{L^{2}(e)^{3}} \leq c\left\|\operatorname{div} \mathbf{u}_{h}\right\|_{L^{2}(K)}
$$

yield that the indicators $\eta_{K}^{0}$ and $\eta_{K}^{1}$ are fully equivalent.

In order to prove an upper bound of the error as a function of the indicators, we follow the approach of [19] and [22], Chapter 2. We first establish the residual equations associated with any solutions $(\mathbf{u}, p)$ of problem (2.3) and $\left(\mathbf{u}_{h}, p_{h}\right)$ of problem (3.2). To this end we observe that, for any $\mathbf{v}$ in $\mathbb{X}(\Omega)$ and $\mathbf{v}_{h}$ in $\mathbb{X}_{h}$,

$$
\begin{aligned}
a(\mathbf{u}, \mathbf{v})+c(\mathbf{u}, \mathbf{u}, \mathbf{v})+b(\mathbf{v}, p)-a\left(\mathbf{u}_{h}, \mathbf{v}\right)-c\left(\mathbf{u}_{h}, \mathbf{u}_{h}, \mathbf{v}\right)-b\left(\mathbf{v}, p_{h}\right) \\
\\
\quad=\left\langle\mathbf{f}, \mathbf{v}-\mathbf{v}_{h}\right\rangle-a\left(\mathbf{u}_{h}, \mathbf{v}-\mathbf{v}_{h}\right)-c\left(\mathbf{u}_{h}, \mathbf{u}_{h}, \mathbf{v}-\mathbf{v}_{h}\right)-b\left(\mathbf{v}-\mathbf{v}_{h}, p_{h}\right) .
\end{aligned}
$$

By integrating by parts the right-hand side on each $K$ in $\mathcal{T}_{h}$, we obtain

$a(\mathbf{u}, \mathbf{v})+c(\mathbf{u}, \mathbf{u}, \mathbf{v})+b(\mathbf{v}, p)-a\left(\mathbf{u}_{h}, \mathbf{v}\right)-c\left(\mathbf{u}_{h}, \mathbf{u}_{h}, \mathbf{v}\right)-b\left(\mathbf{v}, p_{h}\right)=\left\langle\mathbf{f}-\mathbf{f}_{h}, \mathbf{v}-\mathbf{v}_{h}\right\rangle+\left\langle R, \mathbf{v}-\mathbf{v}_{h}\right\rangle$,

where the residual $R$ is given by

$$
\begin{aligned}
\langle R, \mathbf{v}\rangle= & \sum_{K \in \mathcal{T}_{h}}\left(\int _ { K } \left(\mathbf{f}_{h}-\nu \operatorname{curl}\left(\operatorname{curl} \mathbf{u}_{h}\right)+\nu \operatorname{grad}\left(\operatorname{div} \mathbf{u}_{h}\right)\right.\right. \\
& \left.-\left(\operatorname{curl} \mathbf{u}_{h}\right) \times \mathbf{u}_{h}-\operatorname{grad} p_{h}\right)(\mathbf{x}) \cdot \mathbf{v}(\mathbf{x}) \mathrm{d} \mathbf{x} \\
& -\sum_{e \in \mathcal{E}_{K}} \int_{e}\left(\nu\left(\operatorname{curl} \mathbf{u}_{h}\right) \times \mathbf{n}+\nu\left(\operatorname{div} \mathbf{u}_{h}\right) \mathbf{n}-p_{h} \mathbf{n}\right)(\boldsymbol{\tau}) \cdot \mathbf{v}(\boldsymbol{\tau}) \mathrm{d} \boldsymbol{\tau} \\
& \left.-\sum_{e \in \mathcal{E}_{K}^{m}} \int_{e}\left(\nu\left(\operatorname{curl} \mathbf{u}_{h}\right) \times \mathbf{n}\right)(\boldsymbol{\tau}) \cdot \mathbf{v}(\boldsymbol{\tau}) \mathrm{d} \boldsymbol{\tau}\right) .
\end{aligned}
$$

On the other hand, it is readily checked that, for all $q$ in $L^{2}(\Omega)$,

$$
b\left(\mathbf{u}-\mathbf{u}_{h}, q\right)=\int_{\Omega}\left(\operatorname{div} \mathbf{u}_{h}\right)(\mathbf{x}) q(\mathbf{x}) \mathrm{d} \mathbf{x} .
$$

In what follows we consider solutions of problem (2.3) which satisfy a hypothesis weaker than Assumption 4.4:

Assumption 5.1. The solution $(\mathbf{u}, p)$ of problem (2.3) is such that the operator $\operatorname{Id}+\mathcal{S} D \mathcal{G}(\mathbf{u})$ is an isomorphism of $\mathbb{X}(\Omega)$.

Notation 5.2. Let $\mathcal{V}$ be a neighbourhood of the re-entrant corners and edges in $\Gamma_{m}$. For each re-entrant edge $E_{i}$ with angle $\omega_{i}, \pi<\omega_{i}<2 \pi$, we consider a neighbourhood $\mathcal{V}_{i}$ of a part of $E_{i}$ in $\mathcal{V}$ which does not contain any re-entrant corner and does not intersect any other $\mathcal{V}_{j}$. Next, with each $K$ in $\mathcal{T}_{h}$, we associate the quantity $\gamma_{K}$ :

- larger than $1-\frac{\pi}{\omega_{i}}$ if $K$ is contained in $\mathcal{V}_{i}$;

- equal to $\frac{1}{2}$ if $K$ intersects $\mathcal{V}$ but is not contained in any $\mathcal{V}_{i}$;

- equal to 0 otherwise. 
We are now in a position to prove the upper bound for the error. It requires a further assumption on the space $\mathbb{X}_{h}$ that is satisfied by all examples of spaces that we have in mind, and in particular by the spaces $\mathbb{X}_{h}$ introduced in Examples 3.1 and 3.2.

Proposition 5.3. Suppose that the data $\mathbf{f}$ belong to $L^{2}(\Omega)^{3}$, that $\mathbb{X}_{h}$ contains the space

$$
\overline{\mathbb{X}}_{h}=\left\{\mathbf{v}_{h} \in \mathbb{X}(\Omega) ; \forall K \in \mathcal{T}_{h}, \mathbf{v}_{h \mid K} \in \mathcal{P}_{1}(K)^{3}\right\}
$$

and that the solution $(\mathbf{u}, p)$ satisfies Assumption 5.1. Then, there exists a neighbourhood $\mathcal{U}^{*}$ of $\mathbf{u}$ in $\mathbb{X}(\Omega)$ such that any solution $\left(\mathbf{u}_{h}, p_{h}\right)$ of problem (3.2) with $\mathbf{u}_{h}$ in $\mathcal{U}^{*}$ satisfies the following a posteriori error estimate

$$
\left\|\mathbf{u}-\mathbf{u}_{h}\right\|_{\mathbb{X}(\Omega)}+\left\|p-p_{h}\right\|_{L^{2}(\Omega)} \leq c\left(\sum_{K \in \mathcal{T}_{h}} h_{K}^{-2 \gamma_{K}}\left(\left(\eta_{K}^{\varepsilon}\right)^{2}+h_{K}^{2}\left\|\mathbf{f}-\mathbf{f}_{h}\right\|_{L^{2}(K)^{3}}^{2}\right)\right)^{\frac{1}{2}} .
$$

Proof. We only establish estimate (5.5) for $\varepsilon=1$, since it implies the estimate for $\varepsilon=0$ owing to (5.2). It is performed in two steps.

(1) The same arguments as in the proof of Lemma 4.7 combined with inequality (2.8) yield that the mapping $\mathbf{w} \mapsto \operatorname{Id}+\mathcal{S} D \mathcal{G}(\mathbf{w})$ is Lipschitz-continuous from $\mathbb{X}(\Omega)$ into $\mathcal{L}(\mathbb{X}(\Omega))$. Thus, owing to Assumption 5.1, it follows from [19] (see also [22], Prop. 2.1) that, for any $\mathbf{u}_{h}$ in an appropriate neighbourhood $\mathcal{U}^{*}$ of $\mathbf{u}$,

$$
\left\|\mathbf{u}-\mathbf{u}_{h}\right\|_{\mathbb{X}(\Omega)} \leq c\left\|\mathbf{u}_{h}+\mathcal{S G}\left(\mathbf{u}_{h}\right)\right\|_{\mathbb{X}(\Omega)}
$$

From equations (4.3), (5.3) and (5.4), we obtain

$$
\begin{gathered}
\left\|\mathbf{u}-\mathbf{u}_{h}\right\|_{\mathbb{X}(\Omega)} \leq c\left(\sup _{\mathbf{v} \in \mathbb{X}(\Omega)} \inf _{\mathbf{v}_{h} \in \mathbb{X}_{h}} \frac{\left\langle\mathbf{f}-\mathbf{f}_{h}, \mathbf{v}-\mathbf{v}_{h}\right\rangle+\left\langle R, \mathbf{v}-\mathbf{v}_{h}\right\rangle}{\|\mathbf{v}\|_{\mathbb{X}(\Omega)}}\right. \\
\left.+\sup _{q \in L_{0}^{2}(\Omega)} \frac{\int_{\Omega}\left(\operatorname{div} \mathbf{u}_{h}\right)(\mathbf{x}) q(\mathbf{x}) \mathrm{d} \mathbf{x}}{\|q\|_{L^{2}(\Omega)}}\right) .
\end{gathered}
$$

The next steps rely on the existence of a suitable Clément regularization operator mapping $L^{2}(\Omega)^{3}$ into $\mathbb{X}_{h}$. For the standard no-slip boundary conditions this operator is constructed in [5], Theorem IX.3.11 and Corollary IX.3.12. By straightforward but technical arguments this construction can easily be modified to take the present boundary conditions into account. This operator has the following local approximation properties: when $\frac{1}{2}<t \leq 2$, for each $K$ in $\mathcal{T}_{h}$ and each face $e$ of $K$

$$
\begin{aligned}
\left\|\mathbf{v}-\mathcal{C}_{h} \mathbf{v}\right\|_{L^{2}(K)^{3}} & \leq c h_{K}^{t}\|\mathbf{v}\|_{H^{t}\left(\Delta_{K}\right)^{3}} \\
\left\|\mathbf{v}-\mathcal{C}_{h} \mathbf{v}\right\|_{L^{2}(e)^{3}} & \leq c h_{e}^{t-\frac{1}{2}}\|\mathbf{v}\|_{H^{t}\left(\Delta_{e}\right)^{3}}
\end{aligned}
$$

where $\Delta_{K}$ and $\Delta_{e}$ stand for the unions of all elements $K$ of $\mathcal{T}_{h}$ that intersect $K$ and $e$, respectively. On the other hand, by combining the arguments in the proof of Lemma 2.5 with [3], Remark 2.16, we obtain that, for the $\mathcal{V}$ introduced in Notation 5.2, the spaces of restrictions of functions of $\mathbb{X}(\Omega)$ to $\Omega \backslash \overline{\mathcal{V}}$ and to $\mathcal{V}$ are embedded in $H^{1}(\Omega \backslash \overline{\mathcal{V}})^{3}$ and $H^{t}(\mathcal{V})^{3}, t>\frac{1}{2}$ (this follows from [10], equation (2.1) since $\Omega$ is a polyhedron). Moreover, it also follows from [10] that the space of restrictions of functions of $\mathbb{X}(\Omega)$ to $\mathcal{V}_{i}$ for the $\mathcal{V}_{i}$ also introduced in Notation 5.2 are embedded in $H^{s}\left(\mathcal{V}_{i}\right)^{3}$ for all $s<\frac{\pi}{\omega_{i}}$. Hence, taking $\mathbf{v}_{h}$ equal to $\mathcal{C}_{h} \mathbf{v}$ in inequality (5.6) and using estimates (5.7) combined with these embeddings, we obtain the desired estimate for $\left\|\mathbf{u}-\mathbf{u}_{h}\right\|_{\mathbb{X}(\Omega)}$.

(2) By computing $b\left(\mathbf{v}, p-p_{h}\right)$ from equation (5.3), using the inf-sup condition (2.6) and the fact that the norms of both $\mathbf{u}$ and $\mathbf{u}_{h}$ are bounded independently of $h$, we derive from this estimate the bound of $\left\|p-p_{h}\right\|_{L^{2}(\Omega)}$ stated in (5.5). 
Proving the upper bounds for the indicators is standard. Therefore, we only give an abridged proof of the next statement.

Proposition 5.4. Suppose that the data $\mathbf{f}$ belong to $L^{2}(\Omega)^{3}$. Each indicator $\eta_{K}^{\varepsilon}, K \in \mathcal{T}_{h}$, as defined in equation (5.1) satisfies for any solution $\mathbf{u}$ of problem (2.3)

$$
\eta_{K}^{\varepsilon} \leq c\left(\left\|\mathbf{u}-\mathbf{u}_{h}\right\|_{\mathbb{X}\left(\omega_{K}\right)}+\left\|p-p_{h}\right\|_{L^{2}\left(\omega_{K}\right)}+\sum_{\substack{\kappa \in \mathcal{T}_{h} \\ \kappa \subset \omega_{K}}} h_{\kappa}\left\|\mathbf{f}-\mathbf{f}_{h}\right\|_{L^{2}(\kappa)^{3}}\right),
$$

where $\omega_{K}$ stands for the union of elements of $\mathcal{T}_{h}$ that share a least a face with $K$.

Proof. For the same reasons as above, we only give the proof for $\varepsilon=1$. In formula (5.3), we take $\mathbf{v}_{h}$ equal to zero, and we choose successively $\mathbf{v}$ equal to

(1) $\mathbf{v}_{K}$, with

$$
\mathbf{v}_{K}=\left\{\begin{array}{cl}
\left(\mathbf{f}_{h}-\nu \operatorname{curl}\left(\operatorname{curl} \mathbf{u}_{h}\right)+\nu \operatorname{grad}\left(\operatorname{div} \mathbf{u}_{h}\right)\right. & \\
\left.-\left(\operatorname{curl} \mathbf{u}_{h}\right) \times \mathbf{u}_{h}-\operatorname{grad} p_{h}\right) \psi_{K} & \text { on } K, \\
\mathbf{0} & \text { on } \Omega \backslash K,
\end{array}\right.
$$

where $\psi_{K}$ is the bubble function on $K$, equal to the product of the barycentric coordinates associated with the vertices of $K$;

(2) $\mathbf{v}_{e}$ for each $e$ in $\mathcal{E}_{K}$, with

$$
\mathbf{v}_{e}=\left\{\begin{array}{cl}
\mathcal{L}_{e, K}\left(\left[\nu\left(\operatorname{curl} \mathbf{u}_{h}\right) \times \mathbf{n}\right.\right. & \\
\left.\left.\quad+\nu\left(\operatorname{div} \mathbf{u}_{h}\right) \mathbf{n}-p_{h} \mathbf{n}\right]_{e}\right) \psi_{e} & \text { on } K, \\
\mathcal{L}_{e, K^{\prime}}\left(\left[\nu\left(\operatorname{curl} \mathbf{u}_{h}\right) \times \mathbf{n}\right.\right. & \\
\left.\left.\quad+\nu\left(\operatorname{div} \mathbf{u}_{h}\right) \mathbf{n}-p_{h} \mathbf{n}\right]_{e}\right) \psi_{e} & \text { on } K^{\prime}, \\
\mathbf{0} & \text { on } \Omega \backslash\left(K \cup K^{\prime}\right),
\end{array}\right.
$$

where $\psi_{e}$ is the bubble function on $e, K^{\prime}$ denotes the other element of $\mathcal{T}_{h}$ adjacent to $e$ and $\mathcal{L}_{e, \kappa}$ is a lifting operator from $e$ into $\kappa$ constructed by affine transformation from a fixed lifting operator on the reference element;

(3) $\mathbf{v}_{e}^{m}$ for each $e$ in $\mathcal{E}_{K}^{m}$, with

$$
\mathbf{v}_{e}^{m}= \begin{cases}\mathcal{L}_{e, K}\left(\nu\left(\operatorname{curl} \mathbf{u}_{h}\right) \times \mathbf{n}\right) \psi_{e} & \text { on } K \\ \mathbf{0} & \text { on } \Omega \backslash K\end{cases}
$$

with the same notation as above.

Thus, standard inverse inequalities [22], Lemma 3.3 (see also [5], Sect. XI.2) lead to the desired bound for the first three terms of $\eta_{K}^{1}$. Similarly, in formula (5.4), we take $q$ equal to $q_{K}$, with

$$
q_{K}= \begin{cases}\operatorname{div} \mathbf{u}_{h} & \text { on } K \\ 0 & \text { on } \Omega \backslash K\end{cases}
$$

This yields the bound for the fourth term of $\eta_{K}^{1}$.

Estimate (5.8) is fully optimal and the bound for each $\eta_{K}^{\varepsilon}$ is local, in the sense that it only involves the error in a "small" neighbourhood of $K$. In contrast, estimate (5.5) is optimal only when $\Omega$ has neither re-entrant corners nor re-entrant edges in $\Gamma_{m}$. However, the lack of optimality for a general domain only concerns the elements that intersect a neighbourhood of these re-entrant corners or edges. 


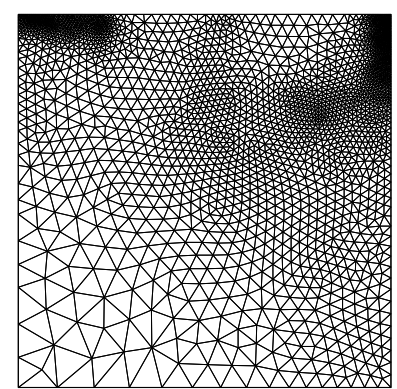

Figure 1. The adapted mesh.
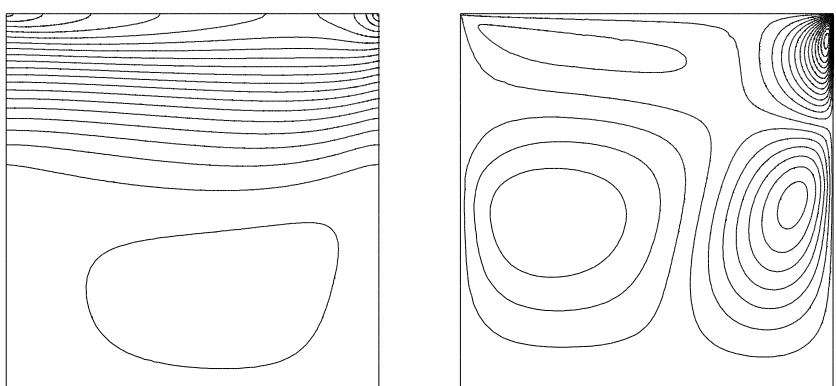

Figure 2. The isovalues of the two components of the velocity.

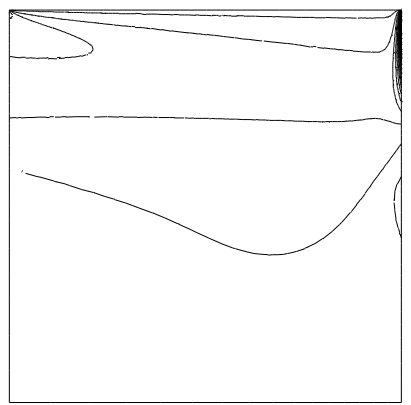

Figure 3 . The isovalues of the vorticity.

\section{A NUMERICAL EXPERIMENT}

The numerical simulation that we now present has been performed on the code FreeFem ++ due to Hecht and Pironneau, see [15]. We only consider a model domain, the square $\Omega=]-1,1\left[^{2}\right.$, and take $\Gamma_{m}$ equal to the upper edge of $\Omega$, namely $\left.\Gamma_{m}=\right]-1,1[\times\{1\}$.

It must be noted that, in dimension 2 , curl $\mathbf{u}$ is a scalar function, so that the condition $(\mathbf{c u r l} \mathbf{u}) \times \mathbf{n}=\mathbf{0}$ on $\Gamma_{m}$ must be replaced by $\mathbf{c u r l} \mathbf{u}=\mathbf{0}$. Up to these modifications, the analysis of the problem and of its discretization is the same as in dimension 3 . We also replace the data $\mathbf{f}$ by a boundary data $\mathbf{g}$ : more precisely $\mathbf{f}$ is equal to zero and the third line in system (1.1) is replaced by

$$
\mathbf{u}=\mathbf{g} \quad \text { on } \Gamma \text {. }
$$


The Navier-Stokes system is solved via the Newton-Raphson method, the initial value of the velocity being computed as the solution of the Stokes problem.

We work with the parameter $\varepsilon$ equal to 1 and with Taylor-Hood finite elements as described in Example 3.2. We take the viscosity $\nu$ equal to $10^{-2}$. The datum $\mathbf{g}$ has its second component equal to zero on $\Gamma$, while its first component $g_{x}$ is given by

$$
\begin{aligned}
& g_{x}( \pm 1, y)= \begin{cases}0, & -1 \leq y \leq 0, \\
y^{2}, & 0 \leq y \leq 1\end{cases} \\
& g_{x}(x,-1)=0,
\end{aligned}
$$

Figure 1 presents the final adapted mesh. It can be noted that $\operatorname{curl} \mathbf{u}_{h}$ is discontinuous at the vertices $( \pm 1,1)$, whence the refinement of the final mesh near these vertices.

Figure 2 presents the curves of isovalues of the two components of the velocity. Figure 3 presents the curve of isovalues of curl $\mathbf{u}_{h}$. The boundary conditions that are enforced on these unknowns are perfectly taken into account in the computation.

\section{REFERENCES}

[1] M. Amara, D. Capatina-Papaghiuc, E. Chacón-Vera and D. Trujillo, Vorticity-velocity-pressure formulation for the Stokes problem. Math. Comput. 73 (2003) 1673-1697.

[2] M. Amara, D. Capatina-Papaghiuc and D. Trujillo, Stabilized finite element method for the Navier-Stokes equations with physical boundary conditions. Math. Comput. 76 (2007) 1195-1217.

[3] C. Amrouche, C. Bernardi, M. Dauge and V. Girault, Vector potentials in three-dimensional nonsmooth domains. Math. Meth. Appl. Sci. 21 (1998) 823-864.

[4] C. Bègue, C. Conca, F. Murat and O. Pironneau, Les équations de Stokes et de Navier-Stokes avec des conditions aux limites sur la pression, in Nonlinear Partial Differential Equations and their Applications, Collège de France Seminar IX, H. Brezis and J.-L. Lions Eds., Pitman (1988) 179-264.

[5] C. Bernardi, Y. Maday and F. Rapetti, Discrétisations variationnelles de problèmes aux limites elliptiques, Mathématiques \& Applications 45. Springer (2004).

[6] F. Brezzi and M. Fortin, Mixed and Hybrid Finite Element Methods, Springer Series in Computational Mathematics 15. Springer, Berlin (1991).

[7] F. Brezzi, J. Rappaz and P.-A. Raviart, Finite-dimensional approximation of nonlinear problems. I. Branches of nonsingular solutions. Numer. Math. 36 (1980/1981) 1-25.

[8] C. Conca, C. Parés, O. Pironneau and M. Thiriet, Navier-Stokes equations with imposed pressure and velocity fluxes. Internat. J. Numer. Methods Fluids 20 (1995) 267-287.

[9] M. Costabel, A remark on the regularity of solutions of Maxwell's equations on Lipschitz domain. Math. Meth. Appl. Sci. 12 (1990) 365-368.

[10] M. Costabel and M. Dauge, Computation of resonance frequencies for Maxwell equations in non smooth domains, in Topics in Computational Wave Propagation, Springer (2004) 125-161.

[11] F. Dubois, Vorticity-velocity-pressure formulation for the Stokes problem. Math. Meth. Appl. Sci. 25 (2002) $1091-1119$.

[12] F. Dubois, M. Salaün and S. Salmon, Vorticity-velocity-pressure and stream function-vorticity formulations for the Stokes problem. J. Math. Pures Appl. 82 (2003) 1395-1451.

[13] K.O. Friedrichs, Differential forms on Riemannian manifolds. Comm. Pure Appl. Math. 8 (1955) 551-590.

[14] V. Girault and P.-A. Raviart, Finite Element Methods for Navier-Stokes Equations, Theory and Algorithms. Springer (1986).

[15] F. Hecht, A. Le Hyaric, K. Ohtsuka and O. Pironneau, Freefem++. Second edition, v. 3.0-1, Université Pierre et Marie Curie, Paris, France (2007), http://www.freefem.org/ff++/ftp/freefem++doc.pdf.

[16] O. Kavian, Introduction à la théorie des points critiques et applications aux problèmes elliptiques, Mathématiques 8 Applications 13. Springer (1993).

[17] J.-L. Lions and E. Magenes, Problèmes aux limites non homogènes et applications 1. Dunod (1968).

[18] M. Orlt and A.-M. Sändig, Regularity of viscous Navier-Stokes flows in nonsmooth domains, in Proc. Conf. Boundary Value Problems and Integral Equations in Nonsmooth Domain, Dekker (1995) 185-201.

[19] J. Pousin and J. Rappaz, Consistency, stability, a priori and a posteriori errors for Petrov-Galerkin methods applied to nonlinear problems. Numer. Math. 69 (1994) 213-231.

[20] S. Salmon, Développement numérique de la formulation tourbillon-vitesse-pression pour le problème de Stokes. Ph.D. Thesis, Université Pierre et Marie Curie, Paris, France (1999).

[21] F. Trèves, Basic Linear Partial Differential Equations. Academic Press (1975).

[22] R. Verfürth, A Review of A Posteriori Error Estimation and Adaptive Mesh-Refinement Techniques. Teubner-Wiley (1996). 